\title{
Root-Secreted Spermine Binds to Bacillus amyloliquefaciens SQR9 Histidine Kinase KinD and Modulates Biofilm Formation
}

\author{
Yunpeng Liu, ${ }^{1}$ Haichao Feng, ${ }^{2}$ Lin Chen, ${ }^{3}$ Huihui Zhang, ${ }^{2}$ Xiaoyan Dong, ${ }^{2}$ Qin Xiong, ${ }^{1}$ \\ and Ruifu Zhang ${ }^{1,2,+}$ \\ ${ }^{1}$ Key Laboratory of Microbial Resources Collection and Preservation, Ministry of Agriculture, Institute of Agricultural Resources \\ and Regional Planning, Chinese Academy of Agricultural Sciences, Beijing, 100081, P.R. China \\ 2 Jiangsu Key Lab and Engineering Center for Solid Organic Waste Utilization, National Engineering Research Center for \\ Organic-based Fertilizers, Nanjing Agricultural University, Nanjing, 210095, P.R. China \\ ${ }^{3}$ Experimental Center of Forestry in North China, Chinese Academy of Forestry, Beijing, 102300, P. R. China
}

Accepted 17 November 2019.

The signal molecules in root exudates that are sensed by plant growth-promoting rhizobacteria (PGPR) are critical to regulate their root colonization. Phosphorylated SpoOA is an important global transcriptional regulator that controls colonization and sporulation in Bacillus species. In this study, we found that deletion of kinD from PGPR strain Bacillus amyloliquefaciens SQR9, encoding an original phosphate donor of Spo0A, resulted in reduced biofilm formation in root exudates compared with the wild-type strain, indicating that KinD is responsible for sensing root exudates. Ligands of B. amyloliquefaciens SQR9 KinD in cucumber root exudates were determined by both the nontargeted ligand fishing method and the targeted surface plasmon resonance detection method. In total, we screened 80 compounds in root exudates for binding to KinD and found that spermine and guanosine could bind to KinD with dissociation constant values of 213 and $51 \mu \mathrm{M}$, respectively. In addition, calcium L-threonate, $N$-acetylL-aspartic acid, sodium decanoic acid, and parabanic acid could also bind weakly to KinD. The three-dimensional binding models were then constructed to demonstrate the interactions between the root-secreted signals and KinD. It was observed that exogenous spermine reduced the wrinkles of biofilm when kinD was deleted, indicating that KinD might be involved in sensing root-secreted spermine and stabilizing biofilm in response to this negative effector. This study provided a new insight of interaction between a rhizobacterial sensor and rootsecreted signals.

Yunpeng Liu and Haichao Feng contributed equally to the article.

${ }^{\dagger}$ Corresponding author: R. Zhang; zhangruifu@caas.cn

Funding: This research was financially supported by the National Natural Science Foundation of China (31600088 and 31700548) and the China Postdoctoral Science Foundation (2016M591297).

*The $\boldsymbol{e}$-Xtra logo stands for "electronic extra" and indicates that supplementary materials are published online.

The author(s) declare no conflict of interest.

๑) 2020 The American Phytopathological Society
Keywords: Bacillus amyloliquefaciens, biocontrol, genetics and gene regulation, root exudates, KinD, interaction, ligands, molecular signaling

Root colonization is the most critical step required for beneficial rhizobacteria to exert their effects on plants (Philippot et al. 2013; Schroth and Hancock 1982). However, the insufficient colonization of functional bacteria on the roots of crops is a common problem for application of microbial inoculums. Root colonization is an integrated behavior that ultimately forms stable biofilms on root surfaces (Belas 2014). Members of genus Bacillus are the most widely used grampositive plant growth-promoting rhizobacteria in agriculture (Lugtenberg and Kamilova 2009). By sensing root exudates, Bacillus species form biofilms on the root or develop spores to resist the disadvantage environment (López and Kolter 2010). How Bacillus species sense root-secreted signals and form biofilms on the root surface is an important topic to solve current problems with the agricultural application of microbial inoculums (López and Kolter 2010).

Biofilm and spore formations of Bacillus species are regarded as two lifestyles of active and inactive mode, respectively, and the switch between them is controlled by spore-forming proteins initiated by the transcription regulator Spo0A (Burbulys et al. 1991; Sonenshein 2000). Spo0A is a critical transcriptional regulator of many processes involved in root colonization, including spore formation, biofilm development, and cannibalism (Boguslawski et al. 2015; González-Pastor 2011; Hamon and Lazazzera 2001). Spo0A is a response regulator, and its activity is controlled by phosphorylation (Fujita and Losick 2005). High phosphorylation of Spo0A leads to spore formation, while low phosphorylation of Spo0A leads to biofilm formation (Mielich-Süss and Lopez 2015). Phosphorylation of Spo0A is mediated by a multicomponent phosphorelay, which comprises SpoOF, Spo0B, and at least four histidine kinases, KinA, KinB, KinC, and KinD (Fujita and Losick 2005; Jiang et al. 2000). These kinases respond to environmental cues, so, in this sense, these kinases-sensed environmental cues provide important variables controlling the colonization of Bacillus species.

The $\mathrm{C}$ termini are similar among these four kinases; however, their $\mathrm{N}$ termini, which are responsible for sensing signals, vary (Fabret et al. 1999). KinD, as the only protein in these kinases 
with an extracellular CACHE domain, was suggested to sense signals from the external environment (Chen et al. 2012; Aguilar et al. 2010). KinD phosphorylated the transcription factor Spo0A at a low level, which induced Bacillus species to form biofilm (Jiang et al. 2000). It has been reported that KinD is required for glycerol and malic acid-induced biofilm formation enhancement (Chen et al. 2012; Morikawa et al. 2006; Shemesh and Chai 2013). KinD from B. subtilis 168 has been found to directly bind pyruvic acid (Wu et al. 2013). However, the root-secreted signals sensed by KinD of Bacillus species have not been comprehensively explored.

Rhizosphere is the area near roots that is rich in nutrients. Plants construct a complex rhizosphere environment by secreting complicated chemical compositions termed root exudates (Dessaux et al. 2016). Root exudates are composed of organic and inorganic compounds. Organic root exudates comprise two different classes of compounds: low molecular weight compounds, including a variety of organic acids, sugars, and secondary metabolites, and high molecular weight compounds, including proteins and polysaccharides (Bais et al. 2006). It is widely accepted that low molecular weight compounds are important signals involved in plant-rhizobacteria interactions (Bais et al. 2006). A strong rhizosphere colonizer should respond to and utilize these root-secreted chemicals efficiently to compete with other microbes in rhizosphere (Ahmad et al. 2011).

Bacillus amyloliquefaciens SQR9 was isolated from the rhizosphere of a cucumber plant and colonized cucumber roots efficiently. In a previous study, we found that root exudates could induce chemotaxis and biofilm formation of B. amyloliquefaciens SQR9 (Zhang et al. 2015). We suggest that B. amyloliquefaciens SQR9 could use KinD as a sensor of plant root exudates, and this hypothesis is confirmed in this study. We further identified the KinD-sensed signals in root exudates by the ligand fishing method and evaluated their effects on biofilm formation in strain SQR9, which revealed that spermine was bound to KinD and reduced wrinkles of SQR9 biofilm when $\mathrm{Kin} D$ was deleted.

\section{RESULTS}

\section{Kinase KinD is involved in biofilm formation of $B$. amyloliquefaciens SQR9 in response to cucumber root exudates.}

KinD is the only Spo0A phosphate donor with an extracellular domain in Bacillus species, so we speculate that the beneficial rhizobacterium $B$. amyloliquefaciens SQR9 might use KinD as the sensor for plant root exudates. To test this speculation in this study, the kinD gene was deleted from B. amyloliquefaciens SQR9 by marker-free chromosome manipulation, and biofilm formation of the mutant and wild-type strain SQR9, in biofilm development basic mineral medium (minimal salts glutamate glycerol [MSgg] medium), was compared in response to root exudates. The concentrations of root exudates were normalized with root fresh weigh. Biofilm formation of the $\operatorname{kin} D$ mutant is reduced, in comparison with the wild-type strain, with root exudates, while no difference was observed between them without root exudates (Fig. 1A). Growth of the wild-type SQR9 and the kinD mutant in the same conditions was measured and did not show a significant difference (Fig. 1B). These results indicated that $k i n D$ is involved in biofilm formation in response to cucumber root exudates.

\section{KinD binds molecules in cucumber root exudates.}

The histidine kinase KinD of B. amyloliquefaciens SQR9 consists of an extracellular domain, two transmembrane regions, and the conserved histidine phosphorylation and ATP hydrolysis domains. The extracellular sensor domain of KinD (KinD-SD) (Lys32-Val243) was cloned, expressed, and purified. Finally, protein samples with a molecular mass of $26.6 \mathrm{kDa}$, more than $90 \%$ purity, and concentration of $1 \mathrm{mg} / \mathrm{ml}$ were obtained for further study (Supplementary Figs. S1 and S2).

Cucumber root exudates were collected, were dried to powder, and were consequently dissolved in buffer to the original concentration determined in the hydroponic culture conditions. The purified KinD and root exudates were used for biolayer interferometry analysis to assess their interactions. The addition of root exudates to the immobilized KinD protein resulted in a clear response of interference, while the change was restored by disassociation. This result indicated that KinD could directly bind to potential ligands in root exudates (Supplementary Fig. S3). However, due to the complicated composition of root exudates, detailed interaction parameters could not be calculated.

\section{Ligand fishing of KinD in the cucumber root exudates.}

To find the potential signal molecules sensed by KinD from the rhizobacterium SQR9 in cucumber root exudates, modified ligand fishing, which is shown in Figure 2, was performed. Briefly, the purified KinD protein and the root exudates were mixed and incubated, and then, the protein-ligand complex was isolated. The protein was denatured to release the binding ligands to the solution. In this experiment, a negative control that lacked KinD-SD protein was included. Subsequent gas chromatography-mass spectrometry (GC-MS) analysis and liquid chromatography (LC)-MS analysis showed visible differences between the spectrum and the aligned peaks (Supplementary Figs. S4, S5, and S6; Supplementary Tables S1, S2 and S3). The relative quantified metabolism analysis identified 15 compounds that potentially bind to KinD, including cytosine, itaconic acid, lactose, $N$-acetyl-L-aspartic acid, pyruvic acid, ornithine, saccharic acid, urea, 2-deoxytetronic acid, 2pyrrolidineacetic acid, and creatine. Among them, nine were identified by GC-MS, while the other six were identified by LC-MS (Table 1; Supplementary Table S4).

\section{Verification of the protein-ligand interaction} by surface plasmon resonance (SPR).

To verify the results from ligand fishing, 10 of the 15 identified molecules were verified for their interactions with KinD, using the SPR method. Along with the potential ligands, 70 other chemicals previously identified in cucumber root exudates were also included (Table 1). All 80 chemicals were tested individually for their interactions with KinD-SD, using the SPR method. The quantification of reaction between the small molecules and KinD-SD was indicated by response unit (RU). One RU equals to $1 \times 10^{-6}$ of refractive index unit, which indicates $1 \mathrm{pg}$ of molecules per square millimeter that bound to the SPR chip through the interaction of the target protein. The binding curves and the parameters were calculated by the double deduction method (Fig. 3A). Results showed that spermine, calcium L-threonate, $\mathrm{N}$-acetyl-L-aspartic acid, sodium decanoic acid, parabanic acid, and guanosine could be bound by KinD-SD (Fig. 3A). Spermine and guanosine showed higher binding affinity than the other four ligands, with dissociation constant $\left(\mathrm{K}_{\mathrm{d}}\right)$ values of 214 and $51 \mu \mathrm{M}$, respectively (Fig. 3B). $K_{a}$ values (equilibrium constant) for spermine and guanosine were $1.14 \times 10^{3} \mathrm{M}^{-1} \mathrm{~s}^{-1}$ and $2.18 \times 10^{4} \mathrm{M}^{-1} \mathrm{~s}^{-1}$, which were higher than the other four ligands (Fig. 3B). Interestingly, our results showed that L-malic acid, glycerol, and pyruvic acid could not bind KinD (data not shown). The $\mathrm{K}_{\mathrm{d}}$ values suggest that spermine and guanosine bind KinD-SD strongly, while calcium L-threonate, $N$-acetyl-L-aspartic acid, sodium decanoic acid, and parabanic acid bind KinD weakly. 


\section{Ligand-KinD docking.}

To better understand the binding model of KinD and ligands, docking analysis was performed. A three-dimensional model of the KinD extracellular domain of B. amyloliquefaciens SQR9 was constructed, using SWISS-MODEL, based on the template from B. subtilis 168 . The natural structure of the KinD extracellular domain is a dimer complex with two typical structures of the CACHE domain. We predicted 62, 43, 24, 25, 14, and 67 models for the interaction of KinD with spermine, calcium Lthreonate, $\mathrm{N}$-acetyl-L-aspartic acid, sodium decanoate, parabanic acid, and guanosine, respectively (Supplementary Table S5). Based on the estimated inhibition constant, the best model for each ligand is shown in Figure 4.

Based on these predicted models, spermine and guanosine showed more negative binding free energy $(-7.1 \mathrm{kcal} / \mathrm{mol})$ in the binding model among these six ligands (Supplementary Table
S5), indicating a higher affinity for KinD-SD that is consistent with the experimental results calculated from the SPR assay (Figs. 3B and 4B). Spermine, guanosine, and parabanic acid formed six, 11, and six hydrogen bonds with KinD-SD, respectively, while the other three ligands did not (Supplementary Table S6). Sodium decanoate, $N$-acetyl-L-aspartic acid, spermine, guanosine, parabanic acid, and calcium L-threonate formed two, one, 12, 11, two, and one polar interactions (Supplementary Table S7) and 13, three, one, two, zero, and one hydrophobic bonds with the KinD, respectively (Supplementary Table S8).

\section{Spermine reduced formation of wrinkles}

in biofilm by the kinD mutant.

Because KinD is involved in regulation of biofilm formation and sporulation, we further studied whether these six compounds affected biofilm formation or sporulation of B. amyloliquefaciens
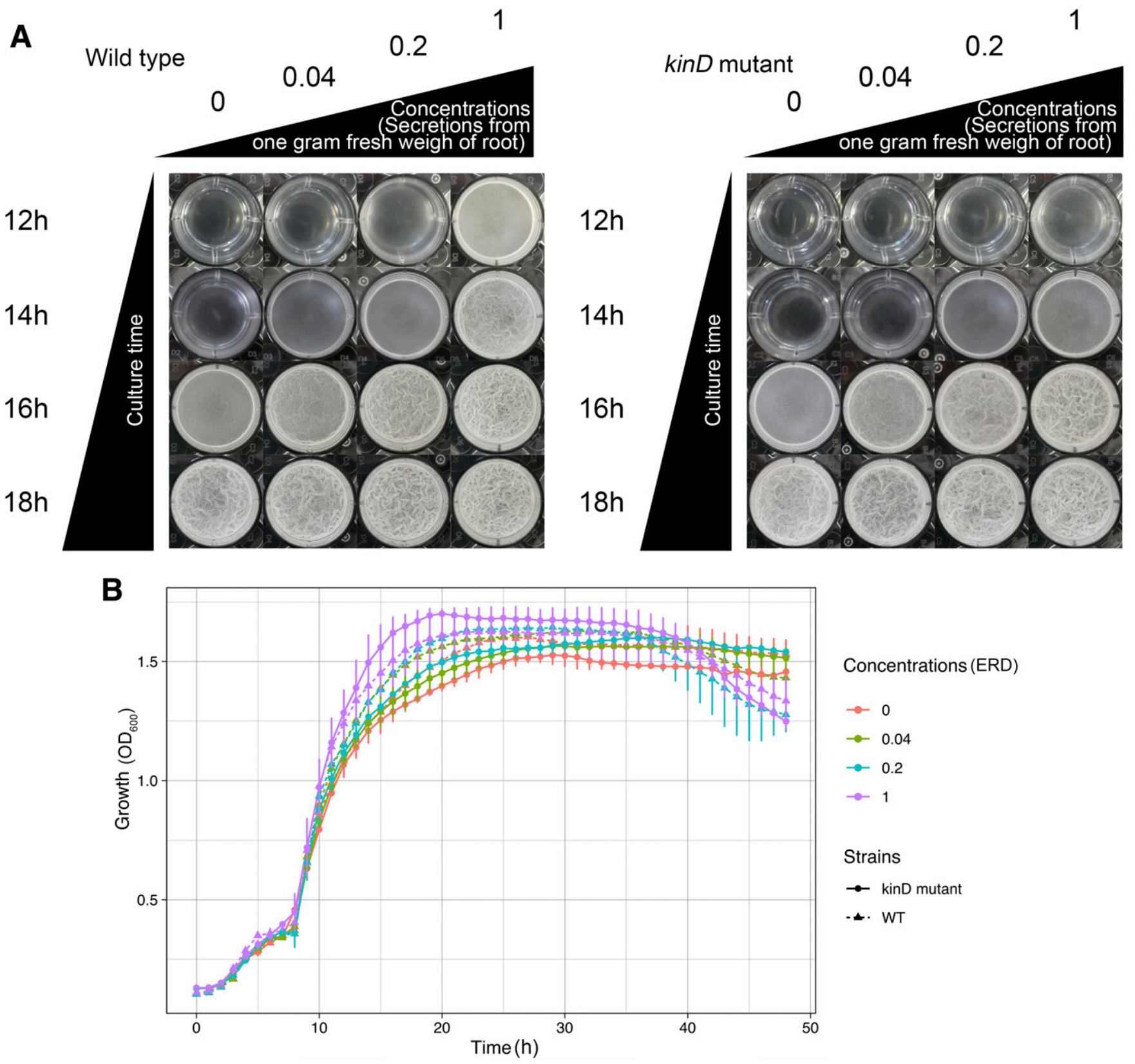

Fig. 1. Biofilm formation of Bacillus amyloliquefaciens SQR9 in response to root exudates. A, A biofilm formation assay was performed with the wild-type strain and the kinD mutant. The amount of root exudates secreted from $1 \mathrm{~g}$ of root fresh weight in 1 day was considered as 1 ERD. Root exudates were added to minimal salts glutamate glycerol medium to the final concentrations of 0.04, 0.2, and $1 \mathrm{ERD} / \mathrm{ml}$. B, Growth curve was measured by Bioscreen C with the same culture conditions as the biofilm formation assay. 
SQR9. These compounds were added in gradient to MSgg medium of SQR9 or the kinD mutant. We did not observe significantly different sporulation between the wild-type strain and the kinD mutant in response to all six compounds (data not shown). However, the biofilm formation in response to spermine was different between them. At 16 to $96 \mathrm{~h}$ postinoculation, $1 \mathrm{mM}$ of spermine dramatically reduced the wrinkles of the biofilm formed by the kinD mutant (Fig. 5A), while no visible difference was observed for the wild-type strain in response to spermine. The growth was further assessed, and the result showed that spermine affected growth of both strains but the kinD mutant did not show different growth compared with wild-type strain (Fig. 5B). These results excluded the possibility that the reduced wrinkles in biofilm formed by kinD mutant was caused by an effect on growth.

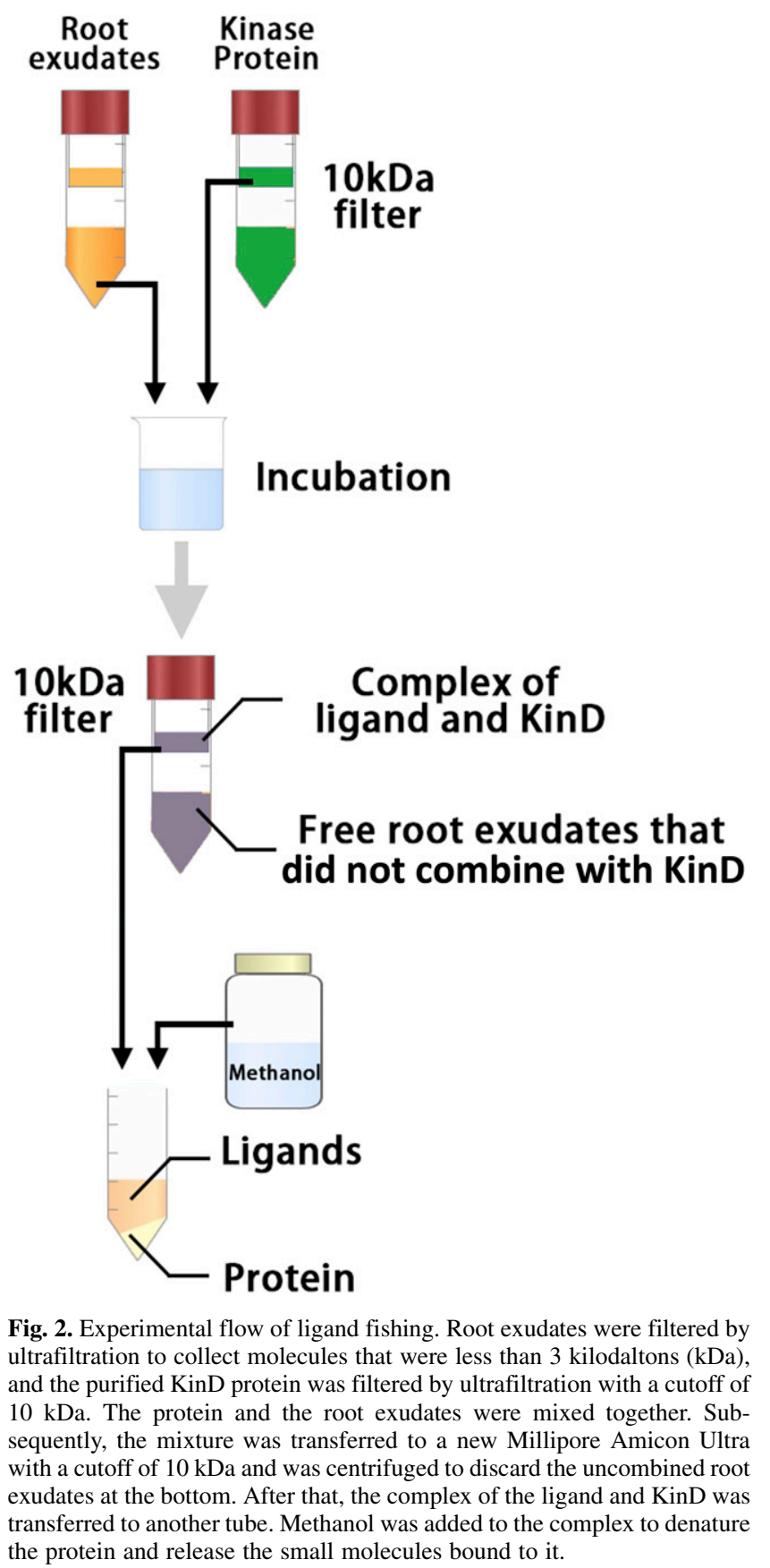

\section{DISCUSSION}

Although root exudates mediate the interactions between beneficial rhizobacteria and their host plants, knowledge of the exact interaction between root exudates and bacterial transmembrane sensors is severely lacking, especially for grampositive bacteria. The Bacillus genus represents the main group of gram-positive bacteria in the rhizosphere (Kumar et al. 2011). KinD is the original phosphate donor of Spo0A, which plays a central role in the lifestyle of Bacillus species (Banse et al. 2011). Although its activity as a kinase has been studied for a long time, the signal in root exudates sensed by KinD is debated. In the present study, by using both nontargeted screening and targeted detection methods, we found six compounds that could be bound by KinD. We also predicted the binding sites and the binding activities of these different ligands, with spermine and guanosine having higher binding affinities for KinD. Moreover, spermine was found to reduce the wrinkles in biofilm formed by the kinD mutant but not the wild-type strain. As the effect of spermine on other bacteria is mostly negative (Goytia et al. 2013; Sobe et al. 2017) while that on Bacillus species is ineffective (Hobley et al. 2017), it is suggested that KinD might be involved in countering the negative effect of spermine on biofilm formation.

A modified ligand fishing method was used in this study (Dixon and Edwards 2010). Although only one compound was confirmed for its binding activity, it showed the possibility of identifying ligands in some complicated cases. In total, 15

Table 1. The compounds in root exudates used for testing the binding activities with the KinD sensor domain (KinD-SD)

Organic acids

Glycolic acid, 3-hydroxypropionic acid, 3-aminoisobutyric acid, 4aminobutyric acid, glyceric acid, $\beta$-glycerolphosphate, parabanic acid, ${ }^{\text {a }}$ benzoic acid, nicotinic acid, glutaric acid, malic acid, salicylic acid, p-hydroxybenzoic acid, phthalic acid, vanillic acid, shikimic acid, azelaic acid, citric acid, lauric acid, myristic acid, pentadecanoic acid, palmitoleic acid, capric acid, stearic acid, threonic acid, pyruvic acid, ${ }^{\mathrm{b}}$ saccharic acid, ${ }^{\mathrm{b}}$ sodium decanoate ${ }^{\mathrm{a}}$

Sugars

Ribose, xylulose, xylose, galactose, mannose, fructose, glucose, tagatose, fucose, sucrose, cellobiose, lactose ${ }^{\mathrm{b}}$ raffinose

Sugar alcohols

Ribitol, galactitol, mannitol, sorbitol, maltotriitol, ribitol, sorbitol, galactinol, maltotriitol

Amino acids

Methionine, L-canavanine, citrullinine, ornithine, ${ }^{\mathrm{b}} \mathrm{N}$-acetylornithine, ${ }^{\mathrm{b}}$ aspartic acid, $N$-acetylL-aspartic acid ${ }^{a, b}$

Other amino compounds

Putrescine, cyclohexylamine, biuret, uracil, nicotinamide, hydroxycarbamate, tyramine, hydroxylamine, $N$-acetyl-Dgalactosamine, guanosine, ${ }^{\mathrm{a}}$ creatine, ${ }^{\mathrm{b}}$ spermidine, spermine, ${ }^{\mathrm{a}}$ cytosine, $^{\mathrm{b}}$ calcium L-threonate ${ }^{\mathrm{a}}$

Others

Urea, ${ }^{\text {b }}$ dihydroxyacetone, acetophenone, myo-inositol, inosine, 1-monopalmitin, 1monostearin, stigmasterol, glycerol $^{\mathrm{b}}$

\footnotetext{
${ }^{a}$ Indicates the compounds with positive binding activity to KinD-SD in surface plasmon resonance experiment.

$\mathrm{b}$ Indicates the compounds identified by the ligand fishing.
} 
compounds were isolated with the ligand fishing method, which indicated the high false-positive rate of the ligand fishing method. It was suggested that the protein added to root exudates would bind the substances with higher contents and higher affinities. As the ligands are not exclusive in root exudates and concentrations vary, the ligand fishing method would show false-negative results for the compounds with low content in root exudates and false-positive results for the compounds with high content. Another interesting idea is that exudates from the bacteria-colonized root instead of normal plant exudates could be a better pool for ligand fishing, because colonization of bacteria could always change the composition of root exudates (Baetz and Martinoia 2014; Liu et al. 2017), but it is important to carefully avoid contamination by bacteria-produced compounds. For many cases, the proteins located on the cell membrane are the first step for sensing signals from other species. As expressing the full-length transmembrane protein is quite a challenge, a truncated protein was always used instead (Feng et al. 2018; Glekas et al. 2010; Webb et al. 2017; Wu et al. 2013). However, a common problem is whether the truncated protein was naturally structured. Improvement of the technique for expressing full-length transmembrane protein would help improve the efficiency of this ligand-fishing method.

Several studies have demonstrated that KinD is involved in Bacillus species to sense root exudates, but the sensed molecules are debated. Shemesh and Chai (2013) demonstrated that the extracellular CACHE domain of $\mathrm{KinD}$ is responsible for sensing the presence of $1 \%$ glycerol ( $\mathrm{vol} / \mathrm{vol})$ and $0.01 \mathrm{mM}$ $\mathrm{MnCl}_{2}$ to stimulate biofilm formation, and the kinD mutant had the most defective phenotype in response to glycerol and manganese. Chen et al. (2012) showed that root exudates of tomato strongly stimulated biofilm formation of B. subtilis, and a small molecule in the exudates, L-malic acid, stimulated biofilm formation at a concentration of 500 to $2,500 \mu \mathrm{M}$ in a KinD CACHE domain-dependent manner. However, they did not show if KinD bind glycerol or L-malic acid directly. Subsequently, Wu et al. (2013) resolved the crystal structure of the extracytoplasmic sensing domain of KinD from B. subtilis 168. It was shown that the sensing domain of KinD did not directly bind L-malic acid. Instead, they showed that KinD was cocrystallized with a pyruvate ligand (Wu et al. 2013). In this study, we also found that neither L-malic acid nor glycerol could be directly bound by KinD. These results suggest that L-malic acid and glycerol are more likely to act as substrates for bacteria and to influence the metabolism, which, in turn, stimulate biofilm formation through the KinD pathway, rather than stimulating biofilm formation by directly binding to KinD. In addition, $B$. amyloliquefaciens SQR9 KinD does not bind pyruvic acid, a ligand of $B$. subtilis $168 \mathrm{KinD}$ (Wu et al. 2013). In $B$. subtilis 168, Phe 133 of KinD is one of the binding sites for pyruvic acid, but this residue in B. amyloliquefaciens SQR9 KinD is Tyr (Supplementary Fig. S7), which may suggest that the different ligand-binding preference of KinD resulted from the variation of the amino acid sequence in the extracellular domain (Supplementary Fig. S7).

$\mathrm{Wu}$ et al. (2013) did not confirm that pyruvate is the physiological ligand of KinD because the addition of pyruvate to the medium did not show an apparent phenotypic difference. In this study, spermine, the polyamine ligand with high affinity to KinD-SD, was found to reduce the wrinkles in biofilm formed by the kinD mutant but not the wild-type strain. Spermine belongs to the polyamines, the functions of which on biofilm have been studied for a long time. Polyamines are found in all living

A
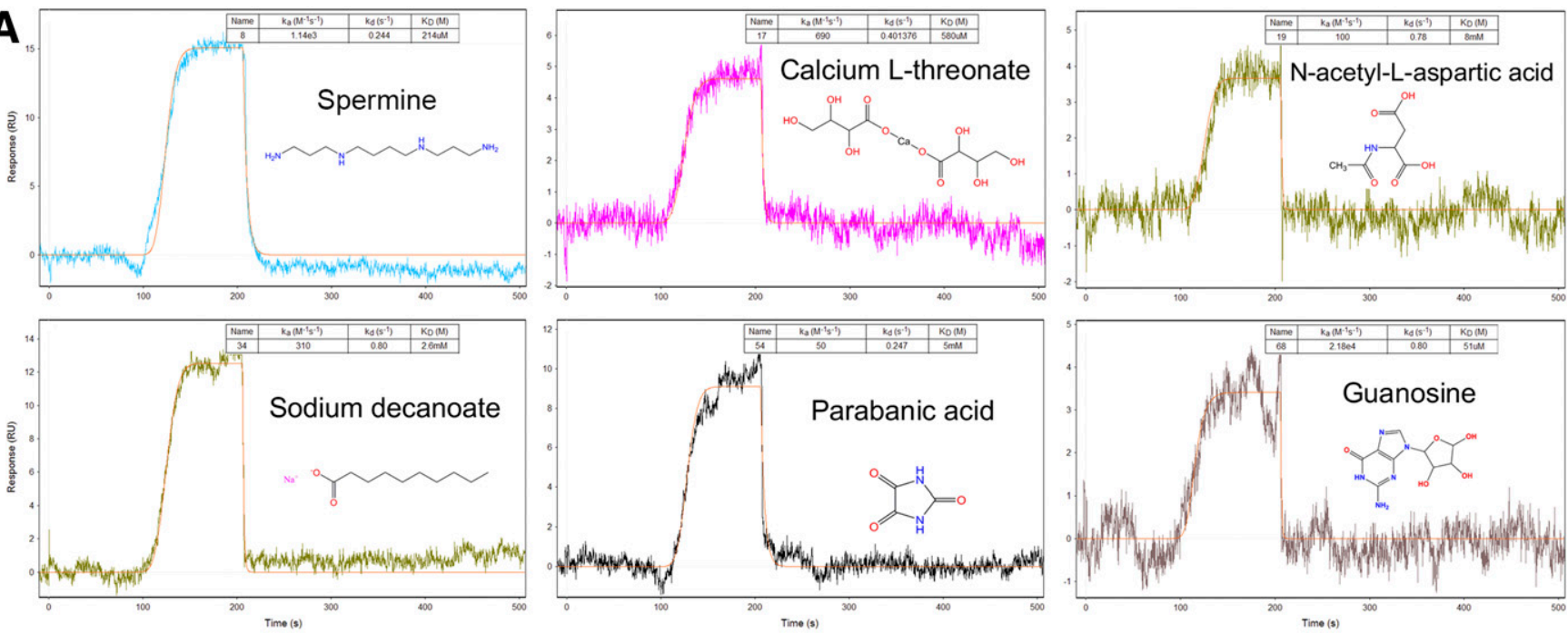

B

\begin{tabular}{llll}
\hline Name & $\mathrm{k}_{\mathrm{a}}\left(\mathrm{M}^{-1} \mathrm{~s}^{-1}\right)$ & $\mathrm{k}_{\mathrm{d}}\left(\mathrm{s}^{-1}\right)$ & $\mathrm{K}_{\mathrm{D}}(\mathrm{M})$ \\
\hline Spermine & $1.14 \times 10^{3}$ & 0.244 & $214 \mu \mathrm{M}$ \\
Calcium L-threonate & 690 & 0.401 & $580 \mu \mathrm{M}$ \\
N-acetyl-L-aspartic acid & 100 & 0.78 & $8 \mathrm{mM}$ \\
Sodium decanoate & 310 & 0.8 & $2.6 \mathrm{mM}$ \\
Parabanic acid & 50 & 0.247 & $5 \mathrm{mM}$ \\
Guanosine & $2.18 \times 10^{4}$ & 0.8 & $51 \mu \mathrm{M}$ \\
\hline
\end{tabular}

Fig. 3. Experimental binding detection by surface plasmon resonance (SPR). A, Binding response of each ligand to the extracellular domain of KinD. B, Kinetic parameter of the binding interaction calculated by Pioneer 4 (v. 2.1.5). The quantification of reaction between the molecules was indicated by response unit (RU). One RU equals to $1 \times 10^{-6}$ of refractive index unit, which indicates $1 \mathrm{pg}$ of molecules per square millimeter that bound to the SPR chip by the interaction with the target protein. 
species, except a few members of Archaea (Thomas and Thomas 2001). Polyamines are critical for regulation of many cell processes, such as growth, stress response, and DNA repair in many organisms (Thomas and Thomas 2001). Spermine inhibits biofilm formation by Neisseria gonorrheae (Goytia et al. 2013) and Vibrio cholerae (Sobe et al. 2017) but has neither inhibition nor promotion effect on B. subtilis (Hobley et al. 2017). We did not observe a difference in biofilm formation by wild-type B. amyloliquefaciens SQR9 in response to spermine, either (Fig. 5). Interestingly, when kinD was deleted, wrinkles of biofilm formed by SQR9 were reduced in response to spermine (Fig. 5). It indicated that root-secreted spermine
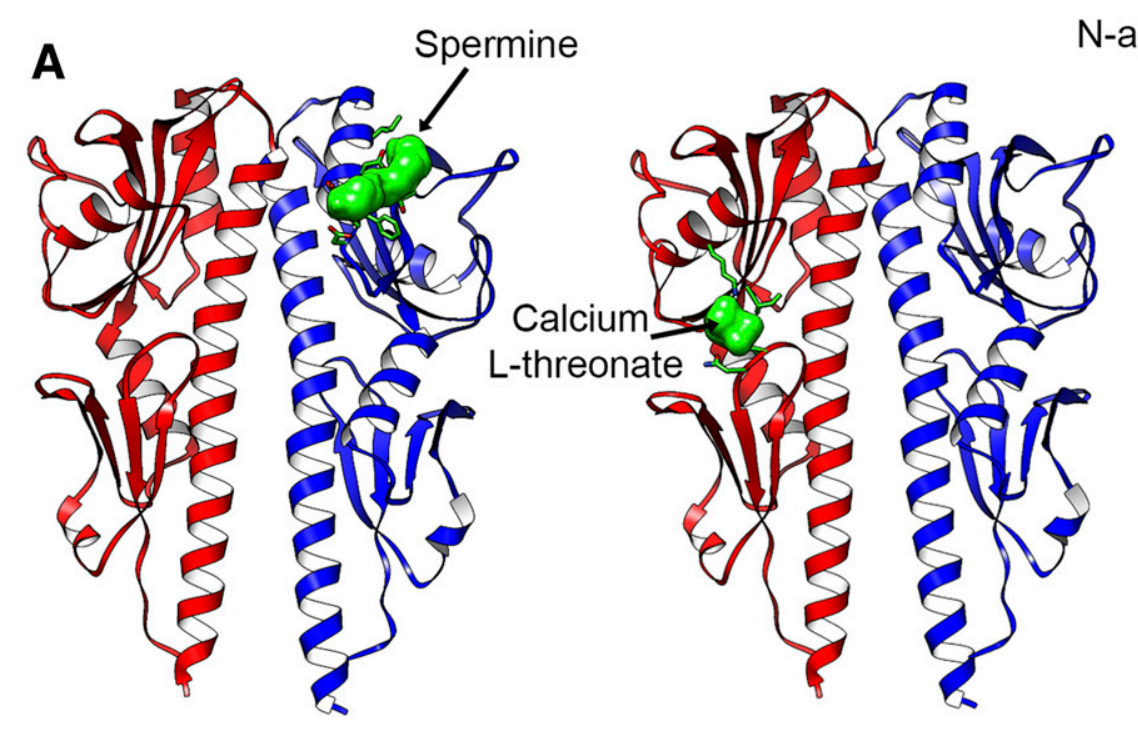

$\mathrm{N}$-acetyl-L-aspartic acid
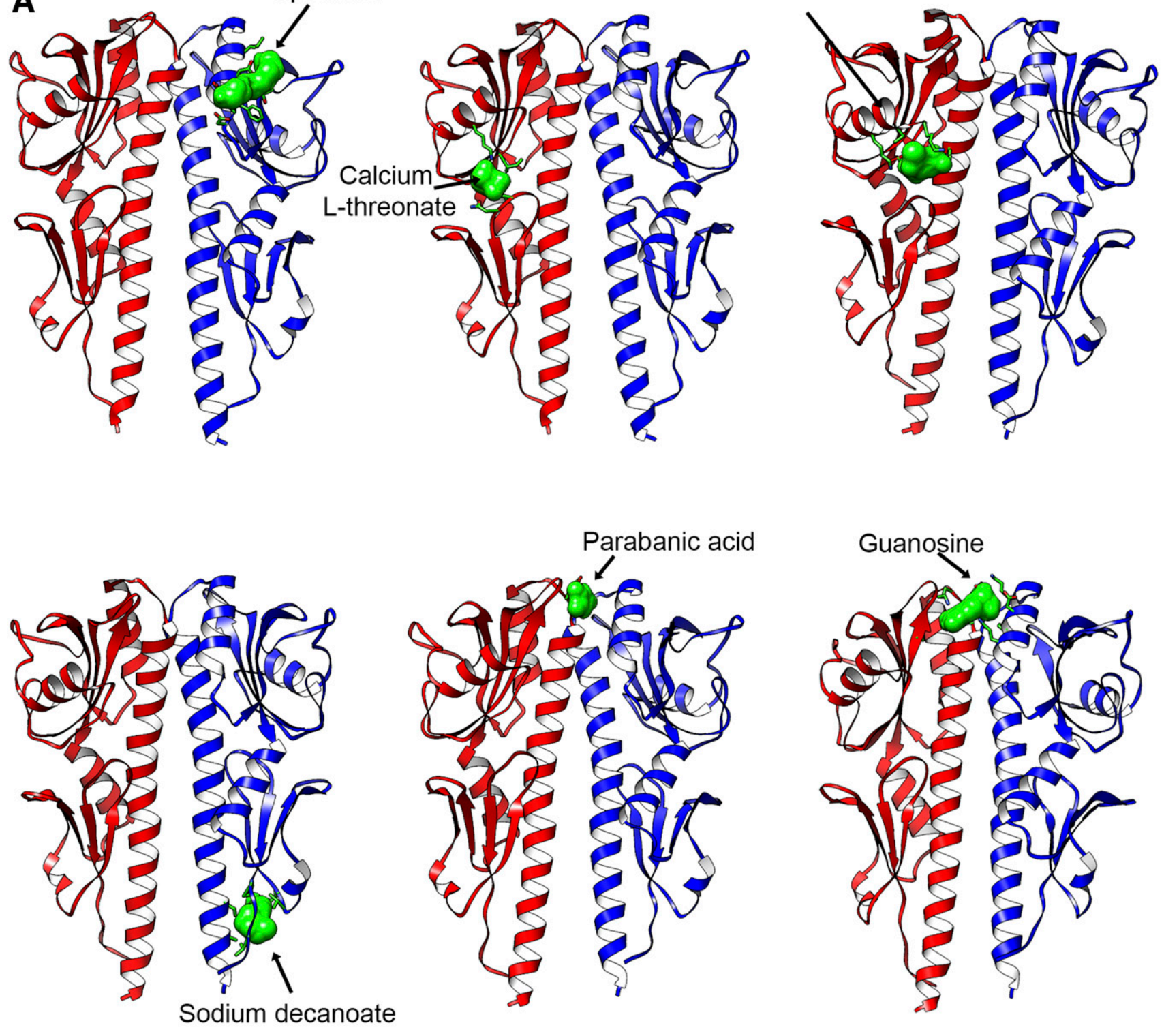

B

\begin{tabular}{lcccccc}
\hline \multicolumn{1}{c}{ Ligands } & $\begin{array}{c}\text { Free Energy } \\
\text { of Binding }\end{array}$ & $\begin{array}{c}\text { Inhibition } \\
\text { Constant, Ki }\end{array}$ & $\begin{array}{c}\text { vdW+Hbond+d } \\
\text { esolv Energy }\end{array}$ & $\begin{array}{c}\text { Electrostatic } \\
\text { Energy }\end{array}$ & $\begin{array}{c}\text { Total Intermolec } \\
\text { Energy }\end{array}$ & $\begin{array}{c}\text { Interact. } \\
\text { Surface }\end{array}$ \\
\hline Spermine & $-7.10 \mathrm{kcal} / \mathrm{mol}$ & $6.29 \mathrm{uM}$ & $-4.95 \mathrm{kcal} / \mathrm{mol}$ & $-6.39 \mathrm{kcal} / \mathrm{mol}$ & $-11.34 \mathrm{kcal} / \mathrm{mol}$ & 507.719 \\
Calcium L-threonate & $-4.07 \mathrm{kcal} / \mathrm{mol}$ & $1.05 \mathrm{mM}$ & $-3.41 \mathrm{kcal} / \mathrm{mol}$ & $-0.24 \mathrm{kcal} / \mathrm{mol}$ & $-3.65 \mathrm{kcal} / \mathrm{mol}$ & 306.783 \\
N-acetyl-L-aspartic acid & $-3.82 \mathrm{kcal} / \mathrm{mol}$ & $1.59 \mathrm{mM}$ & $-2.78 \mathrm{kcal} / \mathrm{mol}$ & $-2.26 \mathrm{kcal} / \mathrm{mol}$ & $-5.04 \mathrm{kcal} / \mathrm{mol}$ & 398.587 \\
Sodium decanoate & $-3.95 \mathrm{kcal} / \mathrm{mol}$ & $1.28 \mathrm{mM}$ & $-5.30 \mathrm{kcal} / \mathrm{mol}$ & $-0.87 \mathrm{kcal} / \mathrm{mol}$ & $-6.17 \mathrm{kcal} / \mathrm{mol}$ & 487.771 \\
Parabanic acid & $-3.91 \mathrm{kcal} / \mathrm{mol}$ & $1.37 \mathrm{mM}$ & $-3.59 \mathrm{kcal} / \mathrm{mol}$ & $-0.32 \mathrm{kcal} / \mathrm{mol}$ & $-3.91 \mathrm{kcal} / \mathrm{mol}$ & 321.195 \\
Guanosine & $-6.27 \mathrm{kcal} / \mathrm{mol}$ & $25.21 \mathrm{uM}$ & $-6.29 \mathrm{kcal} / \mathrm{mol}$ & $-0.06 \mathrm{kcal} / \mathrm{mol}$ & $-6.35 \mathrm{kcal} / \mathrm{mol}$ & 562.975 \\
\hline
\end{tabular}

Fig. 4. Interaction models. A, Molecular docking was performed to show the interaction models between the ligand and extracellular domain of KinD. The KinD dimer structure was constructed based on the template of Bacillus subtilis 168. Both the red and the blue chains indicate the extracellular domain of the dimer. The ligands are shown in sphere mode. B, The summary of the binding energy and the interaction surface. 
has a potential negative role in biofilm formation by SQR9. This result also answered the previous question about why B. amyloliquefaciens $\mathrm{SQR} 9$ produced only spermidine but not spermine (Chen et al. 2017). As we showed in this study, the negative effect of spermine on SQR9 is not exhibited when kinD is present. Because KinD could modulate phosphorylation of Spo0A, which is responsible for regulating biofilm formation and sporulation (Mielich-Süss and Lopez 2015), we proposed that KinD of the Bacillus strain has evolved to sense the plantproduced potential negative effector of biofilm formation, spermine, and transduce the signal to the global transcriptional regulator spo0A, to stabilize the biofilm formation in the
A

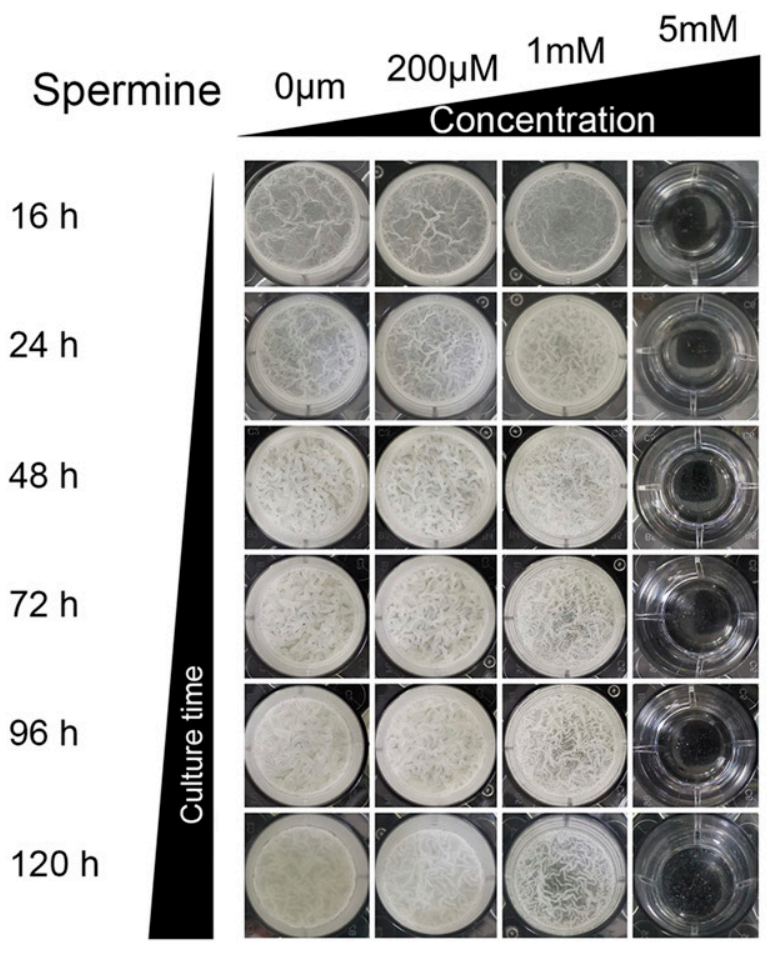

Wild Type

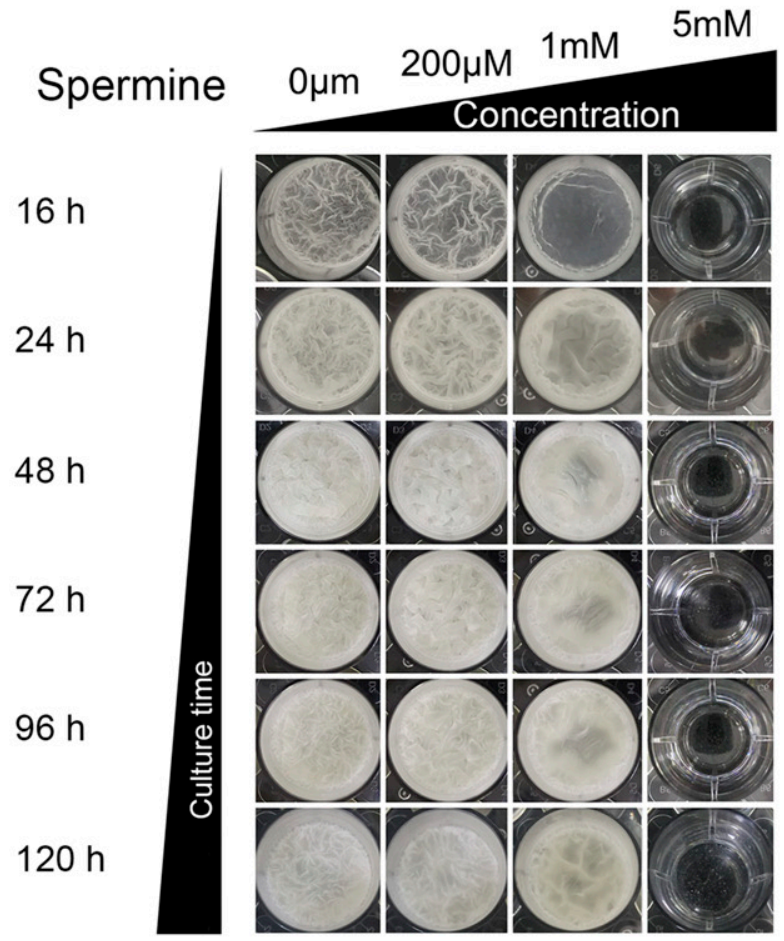

B

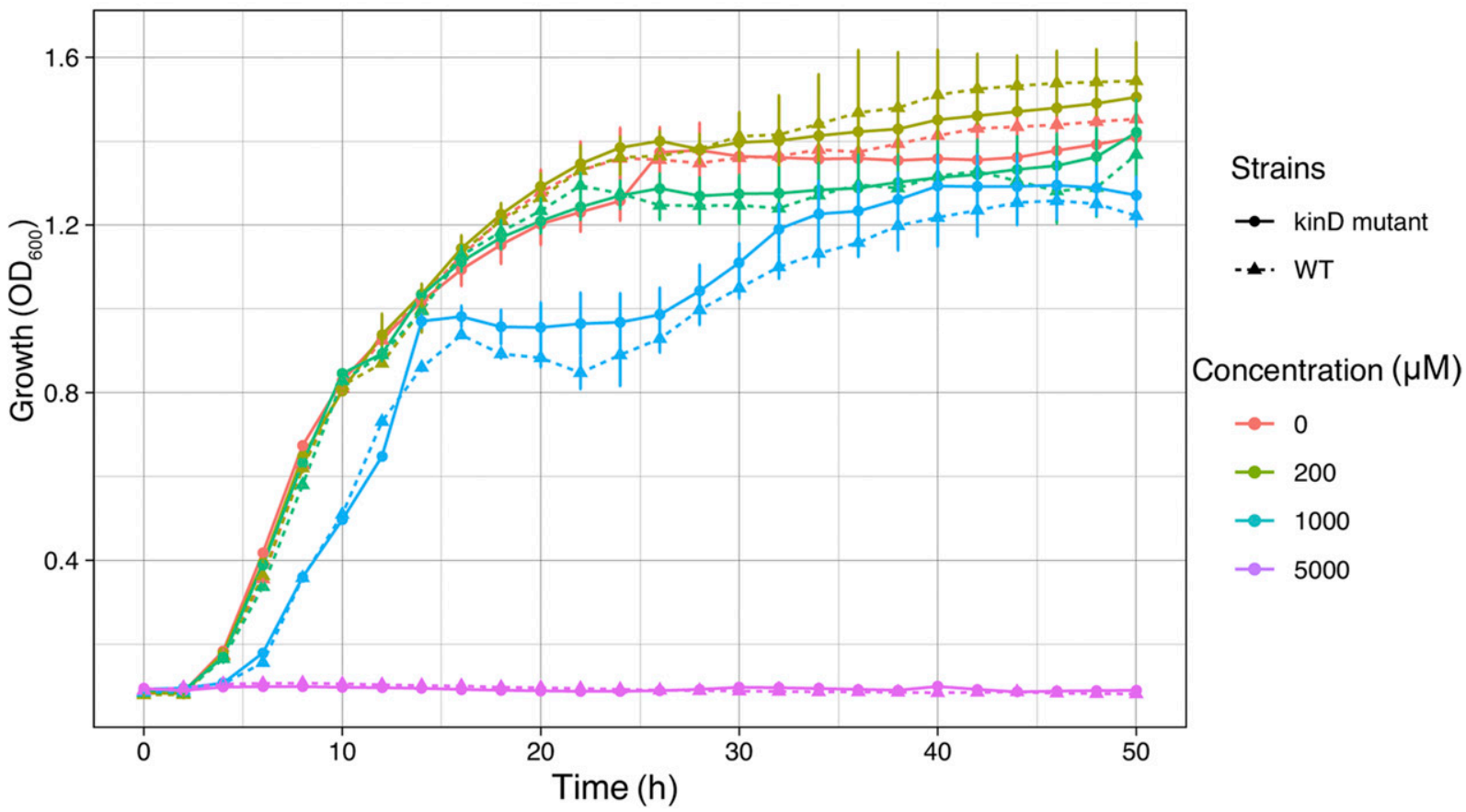

Fig. 5. Biofilm formation of SQR9 in response to spermine. A, Biofilm formation assay was performed with wild-type strain and the kinD mutant. Spermine was added to final concentration of $200,1,000$, or $5,000 \mu \mathrm{M}$, respectively. B, Growth curve was measured by Bioscreen C with the same culture conditions as the biofilm formation assay. 
root-colonizing process. Strikingly, spermine is an important compound in plant tolerance to abiotic stress (Alcázar et al. 2010) and Bacillus species were capable of inducing plant tolerance to abiotic stress; it is tempting to speculate that the sensing of spermine in root exudates by Bacillus species might be associated with the induction of plant systemic tolerance.

In conclusion, six root-secreted compounds were identified as ligands of KinD, the transmembrane sensor of B. amyloliquefaciens SQR9. Among these, spermine reduced the wrinkles of biofilm formed by SQR9 when kinD was deleted, suggesting that KinD might have a function in adaption to the negative effector in rhizosphere.

\section{MATERIALS AND METHODS}

\section{Strains and culture conditions.}

Bacillus amyloliquefaciens SQR9 (China General Microbiology Culture Collection Center accession number 5808) was grown at $37^{\circ} \mathrm{C}$ in Luria-Bertani (LB) medium. Complete deletion of $\operatorname{kin} D$ was performed by marker-free chromosome manipulation, as described previously (Feng et al. 2019).

\section{Biofilm formation assay and growth measurement.}

To determine the effects of the root exudates and the ligands on the biofilm formation of $B$. amyloliquefaciens SQR9, a biofilm formation assay was performed using a 24-well plate, as previously described (Shemesh and Chai 2013). Root exudate concentrations were normalized with the root fresh weigh. Root exudates collected in one day from $0.04,0.2$, or $1 \mathrm{~g}$ of fresh root were dissolved in MSgg medium (5 $\mathrm{mM}$ potassium phosphate [pH 7], $100 \mathrm{mM} 3$-[ $N$-morpholino] propanesulfonic acid [MOPS, pH 7], $2 \mu \mathrm{M} \mathrm{MgCl}, 700 \mu \mathrm{M} \mathrm{CaCl} 2,50 \mu \mathrm{M}$ $\mathrm{MnCl}_{2}, 50 \mu \mathrm{M} \mathrm{FeCl}, 1 \mu \mathrm{M} \mathrm{ZnCl}, 2 \mu \mathrm{M}$ thiamine, $0.5 \%$ glycerol, $0.5 \%$ glutamate, $50 \mu \mathrm{g}$ of tryptophan per milliliter, and $50 \mu \mathrm{g}$ of phenylalanine per milliliter [Branda et al. 2001]), respectively. Concentrations of endogenous spermine in cucumber was present at the micromolar level per gram of dry weight (Fujihara and Yoneyama 2001). In this experiment, spermine was applied at the concentrations around $1 \mathrm{mM}$ (equal to $1 \mu \mathrm{M}$ per gram). Six replicates were included for each treatment.

The growth of the bacteria was indicated by optical density at $600 \mathrm{~nm}\left(\mathrm{OD}_{600}\right)$ and was measured using the Bioscreen $\mathrm{C}$ system. Bacteria were cultured with shaking at $180 \mathrm{rpm}$, while the other culture conditions are same as that used in the biofilm formation assay. Six replicates were included for each treatment.

\section{Protein purification.}

Expression and purification of the extracellular domain of KinD in B. amyloliquefaciens SQR9 was performed. The DNA fragments encoding the extracellular domain (Lys32-Val243) were amplified with primer sets that contained restriction sites for NdeI and HindIII, using SQR9 genomic DNA as a template. The PCR products were digested and cloned into the expression plasmid pET29a(+), which was linearized with the $N d e I$ and HindIII enzymes. The insert regions of the reconstructed plasmid were verified by DNA sequencing. The expressed proteins contained an N-terminal His tag. The constructed expression vectors were individually introduced into Escherichia coli BL21(DE3). The obtained strains were grown in 1-liter Erlenmeyer flasks containing $200 \mathrm{ml}$ of LB medium supplemented with $30 \mathrm{mg}$ of kanamycin per liter at $37^{\circ} \mathrm{C}$ with 200 rpm shaking. When the $\mathrm{OD}_{600}$ of the culture reached 0.6 , the growth temperature was lowered to $16^{\circ} \mathrm{C}$. After another $30-\mathrm{min}$ incubation, the protein expression was induced by adding $0.05 \mathrm{mM}$ isopropyl- $\beta$-D-thiogalactopyranoside. The incubation continued at $16^{\circ} \mathrm{C}$ with 200-rpm shaking overnight, prior to cell harvest by centrifugation at $8,000 \times g$ for $10 \mathrm{~min}$. Then, the cells were washed at least twice with $0.01 \mathrm{M}$ phosphate buffered saline (PBS) buffer ( $\mathrm{pH}$ 7.0). The cell pellets were resuspended in $50 \mathrm{ml}$ of $0.01 \mathrm{M}$ PBS and were disrupted by sonication (2-s pulse on, 3-s pulse off). The cellular lysates were centrifuged at $20,000 \times g$ for $50 \mathrm{~min}$. Finally, for further clarification, the lysates were passed through a $0.22-\mu \mathrm{m}$ filter (Millipore) to remove any other aggregates or insoluble particles, followed by purification with His-affinity resin chromatography according to the manufacturer (Hua Chun) instructions. The purified proteins were collected and stored in PBS buffer at $-80^{\circ} \mathrm{C}$. The purified protein was verified by Fourier transform in cyclotron resonance mass spectrometry (Solarix XR, Bruker).

\section{Root exudate collection.}

Cucumber seeds of cultivar Jinchun 4 were surface-disinfected in a $2 \% \mathrm{NaClO}$ solution for $15 \mathrm{~min}$, were washed thoroughly with distilled water, were planted into axenic tissue culture bottles containing vermiculite, and were allowed to germinate and grow for 4 days in a growth chamber at $23^{\circ} \mathrm{C}$, with a $16-\mathrm{h}$ light and 8-h dark photoperiod. Seedlings were then transplanted into 50-ml flasks containing $35 \mathrm{ml}$ of sterile liquid (one-fourth sucrose-free Murashige Skoog medium) to submerge the seedling roots in the medium, with one seedling in each flask. Seedlings were cultured in these flasks to the three-leaf stage. The Murashige Skoog medium was replaced every other day during the growth period. The hydroponic system was gently shaken $(50 \mathrm{rpm})$ for $2 \mathrm{~h}$ each day on a shaker. All the containers and media were sterilized prior to use. Each device was checked for sterility by taking a 100-ml aliquot and spreading it on solid LB plates; contaminated systems were discarded. For collection of the root exudates, medium was discarded and replaced with sterile double-distilled water. Over the next 2 days, the systems were cultured at $23^{\circ} \mathrm{C}$ with a 16-h light and 8-h dark photoperiod, and the water was collected and replaced every day. Root exudate solutions were filtered through a $0.45-\mathrm{mm}$ membrane (Millipore) and were lyophilized. The sterility of the exudates after filtration was tested by plating $100 \mathrm{ml}$ of each of the root exudates on an LB plate and incubating the plate at $30^{\circ} \mathrm{C}$ to check the contamination. The root parts immersed under the water were cut and dried for weighing.

\section{Biolayer interferometry.}

To generally confirm whether the root exudates held lowmolecular weight signals that bound KinD directly, biolayer interferometry, which indicates the interaction between protein and the small molecules, was performed. Experiments were performed on the Octet-RED 96 device. Data were generated automatically by the Octet User Software (version 9.0). Samples or buffer were dispensed into 96-well black microtiter plates (Millipore) with $200 \mu \mathrm{l}$ in each well. The temperature was set at $25^{\circ} \mathrm{C}$. Ni-NTA biosensor tips (ForteBio, Inc.) were pre-wet with PBS ( $\mathrm{pH}$ 7.0). First, protein samples were immobilized on the biosensor tips by agitation for $10 \mathrm{~min}$ at $1,000 \mathrm{rpm}$; a negative control was included, using biosensor tips immobilized with buffer. Then, the biosensor tips were agitated with root exudate solution for $10 \mathrm{~min}$ at $1,000 \mathrm{rpm}$ for association with the potential ligand; subsequently, the biosensor tips were agitated with PBS for $10 \mathrm{~min}$ at $1,000 \mathrm{rpm}$ for disassociation with the ligand. The binding profile of each sample was summarized as a "nm shift" (the wavelength/spectral shift in nanometers), which represented the association and disassociation of the protein and the potential ligands.

\section{Ligand fishing.}

A modified ligand fishing method was performed (Dixon and Edwards 2010). The exudates from roots of 20 plants that 
secreted for 1 day were dissolved in $5 \mathrm{ml}$ of distilled water. Since we found root exudates could efficiently degrade proteins, inactivation of the proteases in root exudates was performed by boiling them for $30 \mathrm{~min}$ to avoid the degradation of KinD in the following steps. Then, the root exudates were filtered by ultrafiltration to collect the molecules that were smaller than $3 \mathrm{kDa}$ for further use. The purified KinD protein was concentrated to $1 \mathrm{mg} / \mathrm{ml}$ by Millipore Amicon Ultra, with a cutoff of $10 \mathrm{kDa}$. The protein and the root exudates were mixed together with a volume ratio of 1:1 (5 ml for each) and were incubated at $25^{\circ} \mathrm{C}$ for $10 \mathrm{~min}$. Subsequently, the mixture was transferred to a new Millipore Amicon Ultra with a cutoff of $10 \mathrm{kDa}$ and were centrifuged to discard the uncombined root exudates at the bottom. After that, the complex of the ligand and KinD was transferred to another tube. Methanol was added to the complex at a fourfold volume to denature the protein and release the small molecules bound to it. The solution with potential ligands was then injected into GC-MS and LC-MS for isolation and identification.

\section{SPR.}

SPR was performed with the instrument SensiQ (The Pioneer platform, ForteBio) (Kaabinejadian et al. 2016). The Pioneer system is mainly divided into two steps to carry out the experiment. First, for protein immobilization, the SPR chips $(\mathrm{COOH} 5$, ForteBio) were washed with $10 \mathrm{mM}$ $\mathrm{NaOH}$ (flowrate: $25 \mu \mathrm{l} / \mathrm{min}, 2 \mathrm{~min}$; dissociation: $30 \mathrm{~s}$ ) and were then activated with EDC-NHS (0.4 M 1-ethyl-3-[3dimethylaminopropyl] carbodiimide hydrochloride, $0.1 \mathrm{M} \mathrm{N}$ hydroxysuccinimide; flowrate: $10 \mu \mathrm{l} / \mathrm{min}, 10 \mathrm{~min}$; dissociation: $10 \mathrm{~s})$; then, immobilized protein $(50 \mu \mathrm{g} / \mathrm{ml}$, diluted in sodium acetate, $\mathrm{pH}$ 5.0; flowrate: $10 \mu \mathrm{l} / \mathrm{min}, 10 \mathrm{~min}$; dissociation: $10 \mathrm{~s})$ was added, followed by ethanolamine ( $1 \mathrm{M}, \mathrm{pH} 8.0$; flowrate: $10 \mu \mathrm{l} / \mathrm{min}, 5 \mathrm{~min}$; dissociation: $120 \mathrm{~s}$ ) to seal the channel. Second, by using the OneStep injection, the proteinimmobilized chips were allowed to bind the small molecule samples. Binding activities were generated by Qdat matching with the SPR system. The binding signal was indicated by RU value. To measure the binding activity of each compound to KinD-SD, the reaction response between the immobilized chip or the unimmobilized chip and the small molecule or the buffer control were tested. The data were analyzed by Pioneer 4 (V. 2.1.5). The binding curves were calculated by double deduction of the relative controls.

\section{Molecular docking.}

The three-dimensional model of the B. amyloliquefaciens SQR9 KinD extracellular domain (GenBank accession number AHZ15322.1) was constructed, using SWISS-MODEL based on the template from $B$. subtilis 168 . The structure quality checking was performed by SAVES v5.0.

Molecular docking was performed using Dockingserver (Bikadi and Hazai 2009). The MMFF94 force field (Halgren 1996) was used for energy minimization of the ligand molecule (spermine, calcium L-threonate, $\mathrm{N}$-acetyl-L-aspartic acid, sodium decanoate, parabanic acid, or guanosine) using DockingServer. Gasteiger partial charges were added to the ligand atoms. Nonpolar hydrogen atoms were merged and rotatable bonds were defined. Essential hydrogen atoms, Kollman united atom type charges, and solvation parameters were added with the aid of AutoDock tools (Morris et al. 1998). Affinity (grid) maps of $6.3 \times 5.7 \times 7.8 \mathrm{~nm}$ grid points and $0.0375 \mathrm{~nm}$ spacing were generated using the AutoGrid program. AutoDock parameter set- and distance-dependent dielectric functions were used in the calculation of the van der Waals and electrostatic terms, respectively. Docking simulations were performed using the Lamarckian genetic algorithm and the Solis and Wets local search method (Solis and Wets 1981). The initial position, orientation, and torsions of the ligand molecules were set randomly. All rotatable torsions were released during docking. Each docking experiment was derived from 100 different runs that were set to terminate after a maximum of 2,500,000 energy evaluations. The population size was set to 150 . During the search, a translational step of $0.02 \mathrm{~nm}$ and quaternion and torsion steps of five were applied.

\section{AUTHOR-RECOMMENDED INTERNET RESOURCES}

Dockingserver: www.dockingserver.com

SAVES v5.0: https://servicesn.mbi.ucla.edu/SAVES

\section{LITERATURE CITED}

Aguilar, C., Vlamakis, H., Guzman, A., Losick, R., and Kolter, R. 2010. $\mathrm{KinD}$ is a checkpoint protein linking spore formation to extracellularmatrix production in Bacillus subtilis biofilms. MBio 1:e00035-10.

Ahmad, F., Husain, F. M., and Ahmad, I. 2011. Rhizosphere and root colonization by bacterial inoculants and their monitoring methods: a critical area in PGPR research. Pages 363-391 in: Microbes and Microbial Technology. Springer, New York.

Alcázar, R., Altabella, T., Marco, F., Bortolotti, C., Reymond, M., Koncz, C. Carrasco, P., and Tiburcio, A. F. 2010. Polyamines: Molecules with regulatory functions in plant abiotic stress tolerance. Planta 231:1237-1249.

Baetz, U., and Martinoia, E. 2014. Root exudates: The hidden part of plant defense. Trends Plant Sci. 19:90-98.

Bais, H. P., Weir, T. L., Perry, L. G., Gilroy, S., and Vivanco, J. M. 2006. The role of root exudates in rhizosphere interactions with plants and other organisms. Annu. Rev. Plant Biol. 57:233-266.

Banse, A. V., Hobbs, E. C., and Losick, R. 2011. Phosphorylation of Spo0A by the histidine kinase KinD requires the lipoprotein med in Bacillus subtilis. J. Bacteriol. 193:3949-3955.

Belas, R. 2014. Biofilms, flagella, and mechanosensing of surfaces by bacteria. Trends Microbiol. 22:517-527.

Bikadi, Z., and Hazai, E. 2009. Application of the PM6 semi-empirical method to modeling proteins enhances docking accuracy of AutoDock. J. Cheminform. 1:15

Boguslawski, K. M., Hill, P. A., and Griffith, K. L. 2015. Novel mechanisms of controlling the activities of the transcription factors Spo0A and ComA by the plasmid-encoded quorum sensing regulators Rap60-Phr60 in Bacillus subtilis. Mol. Microbiol. 96:325-348.

Branda, S. S., González-Pastor, J. E., Ben-Yehuda, S., Losick, R., and Kolter, R. 2001. Fruiting body formation by Bacillus subtilis. Proc. Natl. Acad. Sci. U.S.A. 98:11621-11626.

Burbulys, D., Trach, K. A., and Hoch, J. A. 1991. Initiation of sporulation in B. subtilis is controlled by a multicomponent phosphorelay. Cell 64: 545-552.

Chen, L., Liu, Y., Wu, G., Zhang, N., Shen, Q., and Zhang, R. 2017. Beneficial rhizobacterium Bacillus amyloliquefaciens SQR9 induces plant salt tolerance through spermidine production. Mol. Plant-Microbe Interact 30:423-432.

Chen, Y., Cao, S., Chai, Y., Clardy, J., Kolter, R., Guo, J. H., and Losick, R. 2012. A Bacillus subtilis sensor kinase involved in triggering biofilm formation on the roots of tomato plants. Mol. Microbiol. 85:418-430.

Dessaux, Y., Grandclément, C., and Faure, D. 2016. Engineering the rhizosphere. Trends Plant Sci. 21:266-278.

Dixon, D. P., and Edwards, R. 2010. Roles for stress-inducible lambda glutathione transferases in flavonoid metabolism in plants as identified by ligand fishing. J. Biol. Chem. 285:36322-36329.

Fabret, C., Feher, V. A., and Hoch, J. A. 1999. Two-component signal transduction in Bacillus subtilis: How one organism sees its world. J. Bacteriol. 181:1975-1983.

Feng, H., Zhang, N., Du, W., Zhang, H., Liu, Y., Fu, R., Shao, J., Zhang, G., Shen, Q., and Zhang, R. 2018. Identification of chemotaxis compounds in root exudates and their sensing chemoreceptors in plant-growthpromoting rhizobacteria Bacillus amyloliquefaciens SQR9. Mol. PlantMicrobe Interact. 31:995-1005.

Feng, H., Zhang, N., Fu, R., Liu, Y., Krell, T., Du, W., Shao, J., Shen, Q., and Zhang, R. 2019. Recognition of dominant attractants by key chemoreceptors mediates recruitment of plant growth-promoting rhizobacteria. Environ. Microbiol. 21:402-415.

Fujihara, S., and Yoneyama, T. 2001. Endogenous levels of polyamines in the organs of cucumber plant (Cucumis sativus) and factors affecting leaf polyamine contents. Physiol. Plant. 113:416-423. 
Fujita, M., and Losick, R. 2005. Evidence that entry into sporulation in Bacillus subtilis is governed by a gradual increase in the level and activity of the master regulator Spo0A. Genes Dev. 19:2236-2244.

Glekas, G. D., Foster, R. M., Cates, J. R., Estrella, J. A., Wawrzyniak, M. J., Rao, C. V., and Ordal, G. W. 2010. A PAS domain binds asparagine in the chemotaxis receptor McpB in Bacillus subtilis. J. Biol. Chem. 285:1870-1878.

González-Pastor, J. E. 2011. Cannibalism: A social behavior in sporulating Bacillus subtilis. FEMS Microbiol. Rev. 35:415-424.

Goytia, M., Dhulipala, V. L., and Shafer, W. M. 2013. Spermine impairs biofilm formation by Neisseria gonorrhoeae. FEMS Microbiol. Lett. 343:64-69.

Halgren, T. A. 1996. Merck molecular force field. I. Basis, form, scope, parameterization, and performance of MMFF94. J. Comput. Chem. 17: 490-519.

Hamon, M. A., and Lazazzera, B. A. 2001. The sporulation transcription factor Spo0A is required for biofilm development in Bacillus subtilis. Mol. Microbiol. 42:1199-1209.

Hobley, L., Li, B., Wood, J. L., Kim, S. H., Naidoo, J., Ferreira, A. S., Khomutov, M., Khomutov, A., Stanley-Wall, N. R., and Michael, A. J. 2017. Spermidine promotes Bacillus subtilis biofilm formation by activating expression of the matrix regulator slrR. J. Biol. Chem. 292: 12041-12053.

Jiang, M., Shao, W., Perego, M., and Hoch, J. A. 2000. Multiple histidine kinases regulate entry into stationary phase and sporulation in Bacillus subtilis. Mol. Microbiol. 38:535-542.

Kaabinejadian, S., McMurtrey, C. P., Kim, S., Jain, R., Bardet, W., Schafer, F. B., Davenport, J. L., Martin, A. D., Diamond, M. S., Weidanz, J. A. Hansen, T. H., and Hildebrand, W. H. 2016. Immunodominant west nile virus $\mathrm{T}$ cell epitopes are fewer in number and fashionably late. J. Immunol. 196:4263-4273.

Kumar, A., Prakash, A., and Johri, B. N. 2011. Bacteria in Agrobiology: Crop Ecosystems. D. K. Maheshwari, ed. Springer, Berlin.

Liu, Y., Chen, L., Wu, G., Feng, H., Zhang, G., Shen, Q., and Zhang, R. 2017. Identification of root-secreted compounds involved in the communication between cucumber, the beneficial Bacillus amyloliquefaciens, and the soil-borne pathogen Fusarium oxysporum. Mol. PlantMicrobe Interact 30:53-62.

López, D., and Kolter, R. 2010. Extracellular signals that define distinct and coexisting cell fates in Bacillus subtilis. FEMS Microbiol. Rev. 34: 134-149.

Lugtenberg, B., and Kamilova, F. 2009. Plant-growth-promoting rhizobacteria. Annu. Rev. Microbiol. 63:541-556.
Mielich-Süss, B., and Lopez, D. 2015. Molecular mechanisms involved in Bacillus subtilis biofilm formation. Environ. Microbiol. 17:555-565.

Morikawa, M., Kagihiro, S., Haruki, M., Takano, K., Branda, S., Kolter, R., and Kanaya, S. 2006. Biofilm formation by a Bacillus subtilis strain that produces $\gamma$-polyglutamate. Microbiology 152:2801-2807.

Morris, G. M., Goodsell, D. S., Halliday, R. S., Huey, R., Hart, W. E., Belew, R. K., and Olson, A. J. 1998. Automated docking using a Lamarckian genetic algorithm and an empirical binding free energy function. J. Comput. Chem. 19:1639-1662.

Philippot, L., Raaijmakers, J. M., Lemanceau, P., and van der Putten, W. H. 2013. Going back to the roots: The microbial ecology of the rhizosphere. Nat. Rev. Microbiol. 11:789-799.

Schroth, M. N., and Hancock, J. G. 1982. Disease-suppressive soil and rootcolonizing bacteria. Science 216:1376-1381.

Shemesh, M., and Chai, Y. 2013. A combination of glycerol and manganese promotes biofilm formation in Bacillus subtilis via histidine kinase KinD signaling. J. Bacteriol. 195:2747-2754.

Sobe, R. C., Bond, W. G., Wotanis, C. K., Zayner, J. P., Burriss, M. A., Fernandez, N., Bruger, E. L., Waters, C. M., Neufeld, H. S., and Karatan, E. 2017. Spermine inhibits Vibrio cholerae biofilm formation through the NspS-MbaA polyamine signaling system. J. Biol. Chem. 292: 17025-17036.

Solis, F. J., and Wets, R. J.-B. 1981. Minimization by random search techniques. Math. Oper. Res. 6:19-30.

Sonenshein, A. L. 2000. Control of sporulation initiation in Bacillus subtilis. Curr. Opin. Microbiol. 3:561-566.

Thomas, T., and Thomas, T. J. 2001. Polyamines in cell growth and cell death: Molecular mechanisms and therapeutic applications. Cell. Mol. Life Sci. 58:244-258.

Webb, B. A., Karl Compton, K., Castañeda Saldaña, R., Arapov, T. D., Keith Ray, W., Helm, R. F., and Scharf, B. E. 2017. Sinorhizobium meliloti chemotaxis to quaternary ammonium compounds is mediated by the chemoreceptor McpX. Mol. Microbiol. 103:333-346.

Wu, R., Gu, M., Wilton, R., Babnigg, G., Kim, Y., Pokkuluri, P. R., Szurmant, H., Joachimiak, A., and Schiffer, M. 2013. Insight into the sporulation phosphorelay: Crystal structure of the sensor domain of Bacillus subtilis histidine kinase, KinD. Protein Sci. 22:564-576.

Zhang, N., Yang, D., Wang, D., Miao, Y., Shao, J., Zhou, X., Xu, Z., Li, Q., Feng, H., Li, S., Shen, Q., and Zhang, R. 2015. Whole transcriptomic analysis of the plant-beneficial rhizobacterium Bacillus amyloliquefaciens SQR9 during enhanced biofilm formation regulated by maize root exudates. BMC Genomics 16:685. 